\title{
Acute effects of exergame-based calisthenics versus traditional calisthenics on state-anxiety levels in young adult men: a randomized trial
}

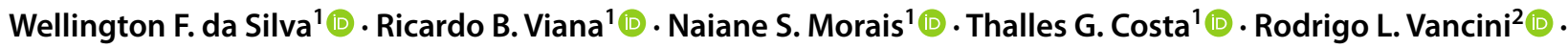

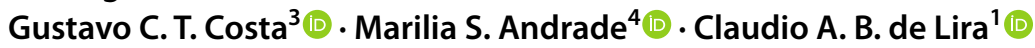

Received: 18 December 2020 / Accepted: 15 September 2021 / Published online: 23 September 2021

(c) The Author(s), under exclusive licence to Springer-Verlag Italia S.r.l., part of Springer Nature 2021

\begin{abstract}
Purpose This study compared the acute effects of an exergame-based calisthenics (EXG) session versus a traditional calisthenics (TC) session on state anxiety levels in healthy adult men, and compared the mean heart rate, number of repetitions performed, enjoyment, and affective valence reported by the participants between sessions.

Methods Thirty-six young adult men performed two 30-min sessions of physical exercise in random order (EXG and TC). The same exercise protocol was used for each session; however, the TC session was guided by an exercise science professional. State anxiety was assessed before and immediately after each session. Heart rate was monitored during the sessions. Enjoyment and affective valence were assessed immediately after the sessions. In addition, the number of repetitions performed was recorded.

Results There was no significant interaction between sessions (EXG vs. TC) and time (pre vs. post-session) $(p=0.102)$, no significant effect of session $(p=0.587)$, and no significant effect of time $(p=0.121)$. Participants presented a higher mean heart rate $(+3.5 \%, p=0.020)$ and number of repetitions performed $(+43.7 \%, p<0.001)$ in the TC session compared with the EXG session. There was no difference in enjoyment $(p=0.804)$ and affective $(p=0.195)$ valence between the EXG and TC sessions.

Conclusion The EXG and TC sessions did not reduce state anxiety levels. Nor did they increase enjoyment or affective valence in healthy young adult men. However, the TC session evoked a higher mean heart rate and higher training volume than the EXG session.
\end{abstract}

Keywords Mental health $\cdot$ Active video games $\cdot$ Exergaming $\cdot$ Exercise $\cdot$ Enjoyment

\section{Introduction}

Adopting a healthier lifestyle and increasing physical activity levels are cornerstones for maintaining physical and mental health $[1,2]$. These behaviors can reduce the risk for the development of mental disorders [2]. Indeed, non-pharmacological treatments, such as regular physical exercise, appear to be useful tools in the prevention and treatment of mental disorders, such as anxiety disorders [3, 4].

According to World Health Organization, 264 million cases of anxiety disorder were documented in 2015, which represents $3.6 \%$ worldwide population [1]. This prevalence is higher in women, who are $60 \%$ more likely than men to experience anxiety disorders over their lifetime [1,5]. However, despite the prevalence of anxiety disorders being higher in women, men are also affected by anxiety disorders 
[1]. In fact, when looking at the prevalence of anxiety disorders in the region of the Americas, this prevalence is 3.9\%, a higher prevalence when compared to other regions (Africa, Europe, Eastern Mediterranean, Southeast Asia and Western Pacific) [1]. Furthermore, when compared to the world average, the prevalence of anxiety in men in the Americas region is $56 \%$ higher compared to the world average [1]. Recently, it was shown in a sample of Brazilian men that $49.1 \%$ of them have anxiety symptoms due to the COVID-19 outbreak [6]. Therefore, strategies for treating anxiety disorders in the male population are also warranted.

Mental disorders place significant social and economic burden on health care systems worldwide, raising the issue of effective and lasting treatments [7]. Anxiety is defined as an emotional state characterized by feelings or emotions of dread, apprehension, and impending disaster; these feelings can develop into maladaptive symptoms and, finally, an anxiety disorder if the symptoms become severe and chronic [1].

Currently, there is evidence of the beneficial effects of physical exercise on mental health [8,9]. Ensari et al. [8], in a meta-analysis, found a small positive effect of acute traditional physical exercise on anxiety disorders compared with no exercise. Another meta-analysis showed that 11 weeks of resistance training ( 2 to 5 days per week) improved anxiety symptoms among healthy participants and participants with a physical or mental illness [9].

Among the physical exercise modalities, exergames (also known as active video games), are innovative and interactive digital games that combine exercise and video gaming $[10,11]$. Exergames appear to be an interesting, pleasant, and enjoyable alternative to traditional exercise methods due to combination of exercise with videogame $[12,13]$. More than a quarter of the world's population is physically inactive [14], and the main arguments for not engaging in an exercise are lack of time, lack of interest, being too tired, lack of motivation, boredom, and not deriving enjoyment from physical exercise [15]. Thus, exergaming may be a useful method of increasing adherence to physical exercise routines. However, despite the increasing interest of the scientific community regarding the potential benefits of exergames, it is reasonable to assume that the population does not use exergames due to the lack of knowledge and unfamiliarity with the tool.

Despite advances in the knowledge on the physiological effects of exergames [12, 16, 17], evidence on the effects of exergames on anxiety disorders has been controversial [13, 18-20]. A recent meta-analysis showed that although exergames provided a significant reduction in anxiety in different clinical populations, the reduction was not greater than that of no exercise [19]. According to the authors, there is great heterogeneity among studies, which could explain the lack of difference in the effects of exergame participation and no exercise [19]. For example, Viana et al. [20] evaluated the effects of a single dance exergame session ( $20 \mathrm{~min})$ at moderate intensity on state anxiety in 40 healthy young adult women. The authors found a significant decrease in state anxiety after the dance exergame session. In contrast, Wagener et al. [18] found no superiority of a dance-based group exergame ("dance pads") over no exercise in reducing anxiety levels in obese adolescents. Thus, it seems that the effects of exergaming on anxiety levels may differ based on exergame protocol and population group.

Some studies have reported that exergames provide a moderate level of physical activity and that mean heart rate ranges from 64 to $76 \%$ of maximal heart rate (HRmax) [13, 17, 20, 21], which corresponds to the recommendations of the American College of Sports Medicine for developing and maintaining cardiorespiratory fitness in apparently healthy adults [22]. Although there are many different exergaming modalities (e.g., cycling, walking, running, climbing stairs, balance training, rowing, swimming, baseball, ping-pong, tennis, boxing, canoeing, stretching exercises, bowling, golf, resistance exercises, yoga, and dancing) [19, 23, 24], little is known about the effects of exergame-based physical exercise on anxiety level compared to non-exergame traditional physical exercise, such as calisthenics exercise.

Calisthenics is a set of physical exercises where only the weight of the body is used to move muscle groups in a natural way, without using dumbbells. Given that calisthenics exercise is widely performed by physical exercise practitioners and that calisthenics exercise is one of the trends of American College of Sports Medicine for fitness [25, 26], studies investigating other forms to practice calisthenics exercise, such as exergamebased calisthenics exercises, can add more information related to the physical exercise prescription for reduce state-anxiety levels. Therefore, the primary purpose of this study was to compare the acute effects of an exergame-based calisthenics (EXG) session with those of a non-exergame traditional calisthenics (TC) session on state anxiety levels in healthy young adult men. We also compared the mean heart rate, number of repetitions performed, enjoyment, and affective valence reported by the participants between sessions. We hypothesized that exergaming would lead to higher improvement in anxiety levels compared with traditional physical exercise. In addition, we hypothesized that exergaming would be more enjoyable and affective and that the results for mean heart rate and number of repetitions performed would be similar to those of the traditional physical exercise intervention.

\section{Methods}

\section{Participants}

The sample size was calculated using $\mathrm{G}^{*}$ Power (version 3.1.9.7; Frans Faul, University of Kiel, Germany). It was 
determined that 35 participants were necessary to achieve a power of $95 \%$ and a $p$-value of 0.05 with a medium effect size (partial eta squared 0.06). Thus, 39 healthy young adult men were recruited, through social media and word of mouth. Inclusion criteria were as follows: (i) men (ii) aged between 18 and 30 years (iii) experience with calisthenics exercise. Exclusion criteria were as follows: (i) contraindications to physical activity (assessed using the Physical Activity Readiness Questionnaire); (ii) diagnosis of mood and/or anxiety disorders; and (iii) use of stimulants (e.g., psychotropic drugs). Two participants were excluded for not meeting the inclusion criteria. One had an illness with repercussions on mood, and the other was contraindicated for physical activity. Another participant discontinued participation in the study due to time constraints (Fig. 1). Therefore, 36 participants $(23.4 \pm 2.9$ years, $78.9 \pm 11.4 \mathrm{~kg}$, $1.77 \pm 0.07 \mathrm{~m}, 25.3 \pm 3.0 \mathrm{~kg} / \mathrm{m}^{2}$, HRmax of $187 \pm 9 \mathrm{bpm}$, and maximal oxygen uptake of $60.9 \pm 6.6 \mathrm{~mL} / \mathrm{kg} / \mathrm{min}$ ) were included in the final analyses. The participants did not have any previous experience with exergames and presented, on average, an intermediate trait anxiety level $(38 \pm 8)$, in accordance with the criteria of Spielberger et al. [27]
All participants were informed about the procedures, and informed consent was obtained before data collection. All procedures were approved by the University Research Ethics Committee (approval number 10067119.6.0000.5083) and were in accordance with the principles outlined in the Declaration of Helsinki.

\section{Study design}

This study used a randomized within-group design. All participants visited the laboratory three times. In the first visit, the participants were administered the Trait subscale of the State-Trait Anxiety Inventory (STAI) [28], were submitted to an anthropometric evaluation and graded maximal exercise testing, and were familiarized with the Xbox 360 Kinect ${ }^{\circledR}$ system (Microsoft Corporation, Redmond, WA, USA). In the second and third visits, participants were randomly assigned to either an exergame-based calisthenics (EXG) session or a traditional calisthenics (TC). The website randomization.com was used to perform the randomization process. State anxiety was assessed before and immediately after each session. Heart rate was monitored during the sessions.
Fig. 1 Flow chart of the study. $T C$ traditional calisthenics

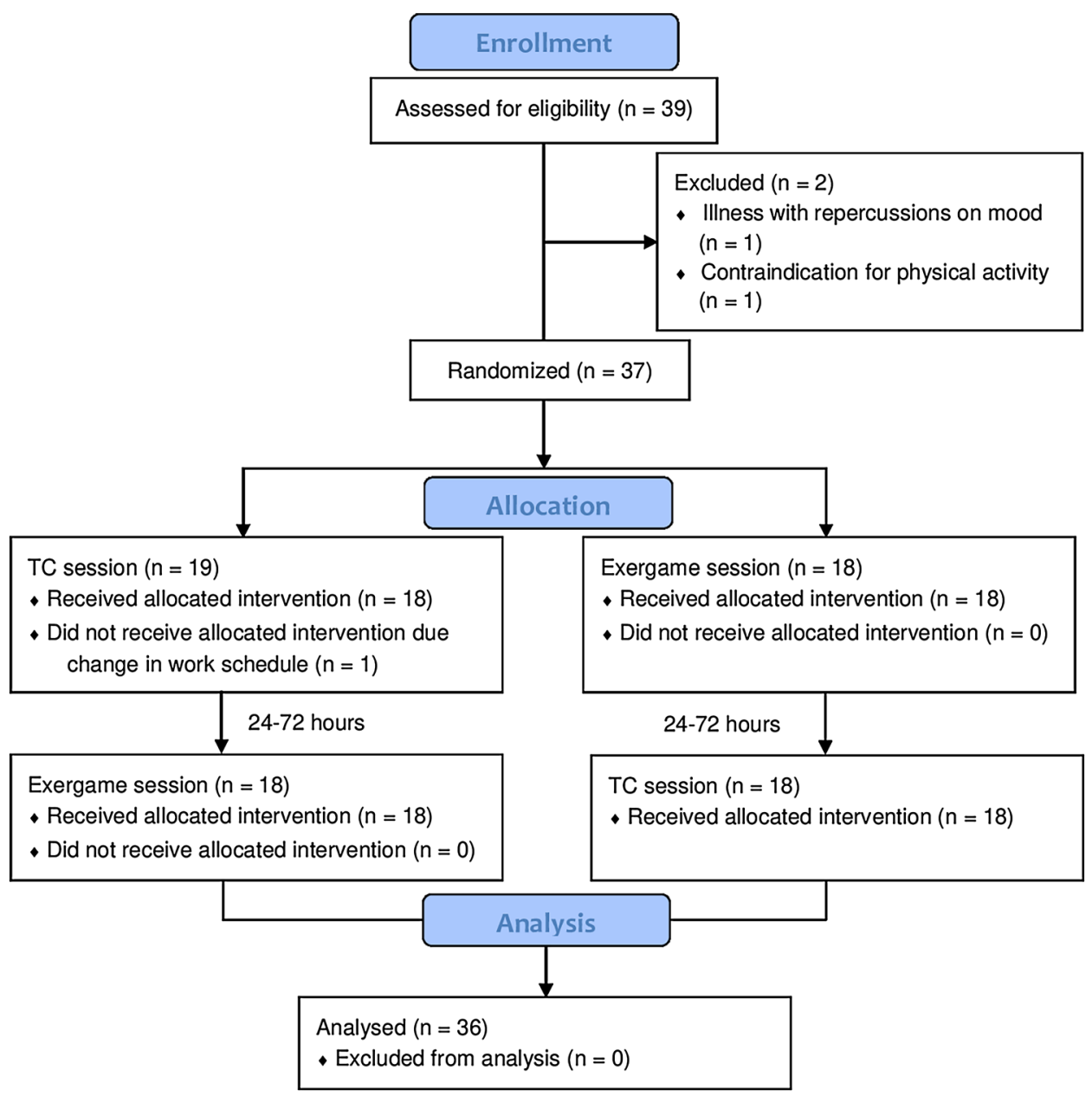


Enjoyment and affective responses were assessed after each session. In addition, the total number of repetitions for each exercise was recorded. Participants received detailed explanation about STAI and enjoyment and affective scales.

All sessions were separated by 24-72 h. The EXG and TC sessions were completed within a 1-week period (Fig. 1). All participants were instructed to refrain from eating $2 \mathrm{~h}$ prior to the sessions and to abstain from caffeine, alcohol, and strenuous physical activity on the day of the experiment. The temperature in the laboratory ranged from 21 to $23^{\circ} \mathrm{C}$. The experiments were conducted in the mornings and afternoons. However, for each participant, the visits were conducted at the same time of the day to respect their circadian rhythm [29]. In addition, participants were advised to maintain the same diet over the study period. Extraneous conversation was minimized during data collection, and the presence of people was restricted to the researchers involved in the study. All experimental procedures were performed individually. There were no medical complications during the experimental procedures and no participant complained of muscle pain after the sessions.

\section{Anthropometric assessment}

Height (in centimeters) and body mass (in $\mathrm{kg}$ ) were measured with a precision of $0.1 \mathrm{~cm}$ and $0.1 \mathrm{~kg}$, respectively. Body mass index was calculated by dividing body mass by height squared $\left(\mathrm{kg} / \mathrm{m}^{2}\right)$.

\section{Graded maximal exercise test}

All participants underwent a graded maximal exercise test on a motorized treadmill (ATL, Inbramed, Porto Alegre, Brazil) with $0 \%$ slope to determine their maximal oxygen uptake and HRmax. The protocol of this test consisted of a 5 -min warm-up period at $7 \mathrm{~km} / \mathrm{h}$, and then the initial speed was progressively increased by $1 \mathrm{~km} / \mathrm{h}$ every minute until exhaustion [30]. During the exercise testing, participants were verbally encouraged to exercise for as long as possible. Respiratory gas samples were measured continuously using a metabolic system (VO2000; MedGraphics, Saint Paul, USA). Prior to testing, the metabolic system was calibrated according to the manufacturer's instructions.

\section{Familiarization}

All participants were given a 5-min introduction to the XBOX 360 Kinect® system to learn how the body movement sensor works. Similar familiarization procedure was used in previous study [20].

\section{Exergame session}

The EXG session lasted approximately $30 \mathrm{~min}$, and the exergame selected was the "Hollywood Workout" (Majesco Entertainment, Edison, Hazlet, NJ, USA). We chose the Sports Athlete protocol because it offers a substantial and varied set of calisthenics exercises performed in exercise facilities (See Supplementary Appendix). The Sports Athlete protocol consists of three phases: "warm-up" (one set of four exercises: jog, trunk crosses, jumping jacks, and ice skaters), "main phase" (three sets of three exercises in a circuit fashion: pushups, skater lunges, and bicycle crunches), and "cool-down" (one set of four exercises: punches, jump rope, side shuffles and mountain climbers). We also chose this exergame protocol because a previous study showed that the exercise intensity elicited is moderate [16], and a moderate intensity level has been found to be associated with health benefits [22]. This training protocol provided a standard total number of repetitions per exercise; however, all participants were instructed to perform the maximum number of repetitions possible within each exercise time (Fig. 2). There was no music for this exergame. Only an audible signal was used to set the start and end of the rest interval between exercises.

\section{Traditional physical exercise session}

The TC session used the same exercise protocol as that of the EXG session. However, there was no video playing on the television, and participants were constantly instructed and guided by a certified exercise professional. The same rest interval between exercises from the EXG session was adopted for the TC session. The same audible signal used

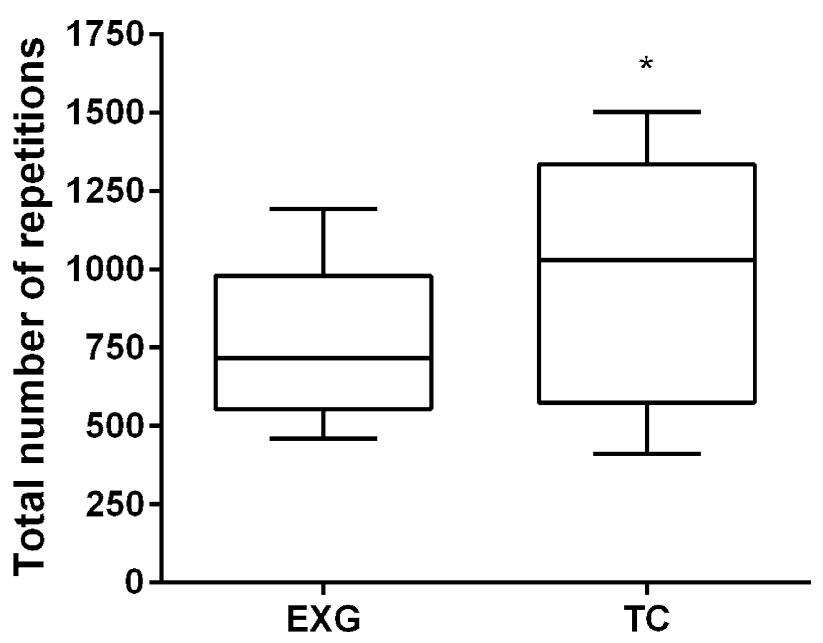

Fig. 2 Total repetitions performed during exergame-based calisthenics (EXG) and traditional calisthenics (TC) sessions $(n=36)$. *Significant difference between exergame-based calisthenics (EXG) and traditional calisthenics (TC) condition $(p<0.001)$ 
during the EXG session was used in the TC session to set the start and end of the rest interval between exercises.

\section{State-trait anxiety assessment}

State anxiety was assessed using the 20-item State subscale of the translated and validated Portuguese version [31] of the STAI [27, 28, 32]. The STAI is a self-report instrument with items on the symptoms and attitudes inherent to anxiety [27, 28, 32]. The STAI contains two subscales: State and Trait. The State and Trait subscales are used to evaluate how the participant feels at the "moment" and "generally," respectively. Items of the STAI are based on a 4-point Likert scale (1 to 4) [27, 28, 32]. Subscale scores ranging from 20 to 30 indicate a low level of state/trait anxiety, 31 to 49 indicate an intermediate level of state/trait anxiety, and 50 or higher indicate a high level of state/trait anxiety [27, 28, 32]. Participants remained seated in a chair inside a private room and the STAI was applied by a trained evaluator. We chose the STAI because of its easy application and low cost, and because it has been used in many different disciplines and translated into 30 languages [27]. Subscale scores range from 20 to 80 points [27, 28, 32]. The Trait subscale was used to characterize the participants. The internal consistency of the State and Trait subscales are good, with Cronbach's alpha of 0.93 and 0.87 , respectively [33].

\section{Heart rate assessment}

Heart rate was continuously monitored with a heart rate monitor (V800, Polar, Kempele, Finland). The monitor's strap was placed across the xiphoid process during the EXG and TC sessions.

\section{Number of repetitions performed}

The participants' training volume in each session (EXG and TC) was determined by the sum of the number of repetitions performed in each exercise. This information was provided by exergames for EXG session and was counted by certified exercise professional for TC session.

\section{Enjoyment and affective valence assessment}

Enjoyment was assessed immediately after the EXG and TC sessions with the Physical Activity Enjoyment Scale (PACES) [34] modified by Graves et al. [24]. The modified PACES retained only five items from the original scale. Individuals rate the extent to which they agree with each item on a 7-point Likert-type scale. Scores range from 5 (no enjoyment at all) to 35 (the highest level of enjoyment) [34]. The PACES score is also displayed as a percentage (\%) of the maximum. Affective valence was assessed immediately after the EXG and TC sessions with the Brazilian Portuguese version [35] of the Feeling Scale [36]. The Feeling Scale is a single-item, 11-point bipolar scale ranging from +5 to -5 , with verbal anchors of "very good" $(+5)$, "good" $(+3)$, "fairly good" $(+1)$, "neutral" (0), "fairly bad" $(-1)$, "bad" $(-3)$, and "very bad" $(-5)$. These tools are acceptable and have already been used in previous studies [13, 20, 37].

\section{Statistical analysis}

Results of the Shapiro-Wilk test indicated that only state anxiety and mean heart rate were normally distributed. Thus, repeated measures analysis of variance with a within-factor of time (time [pre- $\mathrm{x}$ post-session]) was used to compare the state anxiety levels of the EXG and TC sessions. When necessary, Sidak's post hoc test was used to detect differences between means. Student's $t$ test was used to compare EXG and TC sessions in terms of mean heart rate. The Wilcoxon test was used to compare EXG and TC sessions in terms of training volume, enjoyment, and affective valence. The McNemar test was used to compare the proportions of participants with low, intermediate, and high state anxiety levels before and after the EXG and TC sessions. In addition, partial eta squared was used in $\mathrm{G}^{*}$ Power (version 3.1.2) to determine the Cohen's $f$ effect size for analysis of variance (time and session interaction). Then Cohen' $s$ effect size was converted to Cohen's $d$ effect size by the equation $d=f \times 2$ [38]. Cohen's $d$ effect size for the Student's t test was calculated using the equation[39] ${ }_{a}$, where $\Delta, \mathrm{SD}_{\Delta}$, and $r$ represent the mean difference, standard deviation of the mean difference, and coefficient of correlation, respectively. Based on Cohen's guidelines [40], the $d$ values were classified as "trivial" $(d<0.2)$, "small" $(0.2 \leq d<0.5)$, "medium" $(0.5 \leq d<0.8)$, or "large" $(d \geq 0.8)$. The effect size for the Wilcoxon test was calculated using the equation $r_{B}$, [41] where $r_{B}, Z$, and $N$ represent the rank-biserial correlation, Z-score, and sample size, respectively. Classification of $r_{\mathrm{B}}$ values was based on the Pearson's correlation $(r)$ coefficient: "trivial" $\left(r_{\mathrm{B}}<0.10\right)$, "small" $\left(0.10 \leq r_{\mathrm{B}}<0.30\right)$, "medium" $\left(0.30 \leq r_{\mathrm{B}}<0.50\right)$, and "large" $\left(r_{\mathrm{B}} \geq 0.50\right)$ [42]. A significance level of 0.05 was set for all statistical tests. All data were analyzed using the Statistical Package for the Social Sciences (SPSS, version 20.0, IBM Corp., Armonk, NY, USA). Data are presented as mean \pm standard deviation or median and interquartile range (IQR), changes $(\Delta)$ and $95 \%$ confidence interval, and absolute and relative frequencies.

\section{Results}

The EXG and TC sessions lasted $29.8 \pm 1.3 \mathrm{~min}$ and $30.0 \pm 0.7 \mathrm{~min}$, respectively (median 0.28 [IQR: 1.13]; $Z=1.646 ; p=0.100 ; r_{\mathrm{B}}=0.27$ [small]). The EXG total time 
session varied according to the ability of the participants in the transitions between exercises.

\section{State anxiety}

There was no significant interaction between sessions (EXG vs. TC) and time (pre- vs. post-session) $(F[1,35]=2.825$; $p=0.102 ; d=0.57$ [medium]), no main session effect $(F[1,35]=0.300 ; p=0.587)$, and no main time effect $(F[1,35]=2.521 ; p=0.121)$ on state anxiety level (Fig. 3$)$. There was a significant increase in the proportion of participants with intermediate state anxiety levels after the EXG session but not after the TC session (Table 1).

\section{Heart rate}

Participant heart rate was $3.5 \%$ higher in the TC session than in the EXG session ( $\Delta: 4 \mathrm{bpm}$ [95\% CI $1-8 \mathrm{bpm}$ ]; $p=0.020 ; d=0.39$ [small]). Mean heart rates for the TC $(147 \pm 11 \mathrm{bpm})$ and EXG $(143 \pm 12 \mathrm{bpm})$ sessions were $74.7 \pm 5.4 \%$ and $72.4 \pm 5.9 \%$ of HRmax, respectively.

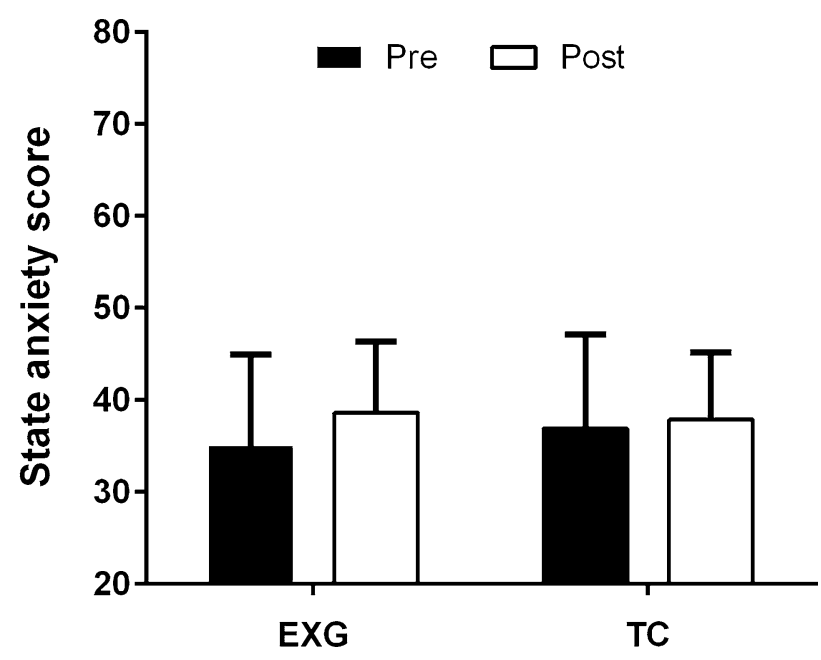

Fig. 3 Participants' anxiety levels before and after exergame-based calisthenics (EXG) and traditional calisthenics (TC) sessions $(n=36)$. There was no significant time and session interaction $(p=0.102)$, no main time effect $(p=0.121)$, and no main session effect $(p=0.587)$

\section{Number of repetitions performed}

The total number of repetitions performed by the participants in the TC session (median 1029 [IQR: 416]) was 43.7\% higher than that (716 [IQR: 103]) in the EXG session ( $\Delta$ : 286 [IQR: 364]; $Z=4.650 ; p<0.001 ; r_{\mathrm{B}}=0.78$ [large]).

\section{Enjoyment and affective valence}

Participants reported similar levels of enjoyment (median 0 [IQR: 4.8]; $Z=0.248 ; p=0.804 ; r_{\mathrm{B}}=0.04$ [trivial]) and affective valence (median 0 [IQR: 1.0 ]; $Z=1.297$; $p=0.195 ; r_{\mathrm{B}}=0.22$ [small]) for both sessions. Overall, participants reported "high" enjoyment (median 27.5 [IQR:10] vs. median 28.5 [IQR:10]) and "good" affective valence (median 3 [IQR:2] vs. median 3 [IQR:3]) for both sessions. Participant enjoyment scores for the TC and EXG sessions were $78.3 \pm 16.0 \%$ and $77.5 \pm 15.7 \%$, respectively.

\section{Discussion}

The present study aimed to compare the acute effects of an exergame-based calisthenics exercise session versus a traditional physical exercise session on anxiety state level, heart rate, training volume, enjoyment, and affective valence in healthy young adult men. Our initial hypothesis was that EXG would evoke a superior anxiolytic effect to that of TC. We also hypothesized that exergame-based physical exercise would be more enjoyable and affective, and would evoke a similar mean heart rate and training volume of the traditional physical exercise session. Our results did not confirm our primary hypothesis, as we did not find an anxiolytic effect in the EXG or TC sessions. Our results also did not confirm our secondary hypothesis; however, the EXG session elicited a similar enjoyment and affective valence. There was a lower mean heart rate for the EXG session, compared with the TC session, and participants performed lower number of repetitions in the EXG session than in the TC session.

Changes in state anxiety level after EXG and TC sessions may be explained by the participants' trait anxiety levels and their state anxiety levels before both sessions. In our sample, $88.9 \%$ of the participants had a trait anxiety level classified as low or intermediate $(91.7 \%$ and $86.7 \%$
Table 1 Participants' state anxiety level classification before and after EXG and TC sessions $(n=36)$

\begin{tabular}{|c|c|c|c|c|c|c|}
\hline & \multicolumn{2}{|l|}{ EXG } & \multirow[t]{2}{*}{$p$} & \multicolumn{2}{|l|}{ TC } & \multirow[t]{2}{*}{$p$} \\
\hline & Pre & Post & & Pre & Post & \\
\hline High level & $8.3 \%(n=3)$ & $13.9 \%(n=5)$ & 0.034 & $13.9 \%(n=5)$ & $8.3 \%(n=3)$ & 0.189 \\
\hline Intermediate level & $55.6 \%(n=20)$ & $69.4 \%(n=25)$ & & $63.9 \%(n=23)$ & $80.6 \%(n=29)$ & \\
\hline Low level & $36.1 \%(n=13)$ & $16.7 \%(n=6)$ & & $22.2 \%(n=8)$ & $11.1 \%(n=4)$ & \\
\hline
\end{tabular}

$E X G$ exergame-based physical exercise, $T C$ traditional calisthenics. P-value from McNemar test 
in the EXG and TC sessions, respectively) with an initial state anxiety level classified as low or intermediate level. Surprisingly, we found a significant increase in the proportion of participants with intermediate state anxiety levels only after the EXG session. However, for both sessions, mean state anxiety scores remained the same, between 31 and 49 points (at pre-and post-sessions), which indicates an intermediate level of state anxiety. These results may be due to a "floor effect," which occurs when an individual's score is at the lowest level of the measure and, therefore, it may impair the detection of changes [8]. Indeed, some studies have failed to find an anxiolytic effect of acute exercise in healthy participants [8], which seems to be related to a low baseline state anxiety level. In addition, the overall high cardiorespiratory fitness of the participants evaluated in the present study $(60.9 \mathrm{~mL} / \mathrm{kg} / \mathrm{min})$ might also have influenced the identification of anxiolytic effects after EXG and TC sessions. Adults who regularly engage in physical exercise may experience fewer symptoms of anxiety, and physical exercise may offer a protective effect against the development of mental disorders [43].

Participants reported high and similar enjoyment after EXG $(77.5 \%)$ and TC (78.3\%) sessions. This result is similar to those found in a previous study [24], which used the same enjoyment scale (modified PACES) adopted in our study. The authors found that adolescents, young adults, and older adults reported an enjoyment of $77.8 \%, 74.7 \%$, and $80.0 \%$, respectively, for Wii muscle conditioning exercises. Our results are exuberant given that enjoyment is an important predictor of participation in regular physical activity [44]. Indeed, it is known that enjoyment is an important factor to increase adherence and satisfaction in the act of doing certain activity [45]. Therefore, TC can be an alternative to other forms of traditional exercise, such as walking, running and resistance training, to increase adherence to an exercise program.

In our study, participants reported "good" affective valence in the EXG and TC sessions. Affective valence is mainly generated by a cognitive assessment process that may involve factors such as self-efficacy, perceived benefits and focus of attention [46, 47]. Several studies have observed that affective responses to physical exercise are dependent on exercise intensity [48, 49]. The personal characteristics of the participants, self-efficacy to carry out interventions and tolerance to the type of exercise proposed can be examples of aspects that explain the results found in relation to the affective valence, as participants with a preference for intense exercise may report a greater affective valence [47]. Again, TC can also be an alternative to increase affective related to exercise.

The sports athlete exercise protocol, from the exergame "Hollywood Workout," places considerable emphasis on calisthenic exercises, which involve the use of the individual's own body mass. This allows the measurement of training volume through the total number of repetitions over an exercise session. Results indicate that the total number of repetitions performed by the participants in the TC session was $43.7 \%$ higher than those in the EXG session. This difference may be due to the exercise 'supervision' by avatar in EXG session. In another words, participants tend to follow the avatar during exergame session. On the other hand, in TC session, the participants did not receive 'supervision'.

Participants' average heart rate during the EXG session (143 bpm [72.4\% of HRmax]) was significantly lower than that during the TC session (147 bpm [74.7\% of HRmax]). This result may be due to the higher total number of repetitions in the TC than in the EXG session. However, the difference between participants' average heart rate corresponded to $4 \mathrm{bpm}$ (small effect size), which in practical terms does not reflect change in exercise intensity. These results can be explained because each exercise lasted approximately $70 \mathrm{~s}$ with rest interval between then; therefore, there was a period of recovery. Furthermore, seminal exercise physiology findings showed that heart rate needs $2-4 \mathrm{~min}$ to adjust for a new value due to metabolic demand [50]; therefore, $70 \mathrm{~s}$ is not sufficient to reveal substantial differences in heart rate between sessions. Based on the criteria set forth by the American College of Sports Medicine [22], these values indicate moderate-intensity exercise. One previous study found similar exergame intensity using the same exergame protocol used in the present study (mean heart rate corresponding to $70.7 \%$ of participants' HRmax) [16]. Moreover, similar results have been found for dance-based exergames in young adults (mean heart rate corresponding to $~ 70 \%$ of participants' HRmax)[51] and healthy women (mean heart rate corresponding to $\sim 70 \%$ of participants' predicted HRmax) [20], and for an exergame controlled only by movement of the lower limbs, in healthy individuals (mean heart rate corresponding to $\sim 72 \%$ of participants' HRmax) [52]. Thus, our results are in line with the literature.

Our study had some limitations. First, the absence of a non-exercise control group increased the risk of random errors. However, we chose to use the TC session as a control group because a quiet rest may serve as an imposed bout of sedentary behavior that acutely may worsen mood states in some individuals [53]. Second, as is the case for all studies that use questionnaires, the current results are based on the honesty of the interviewees. Third, we evaluated only healthy men; therefore, our results may not be generalizable to women and/or clinical populations diagnosed with anxiety disorders. Fourth, participants had no diet control; however, they were advised to maintain the same diet over the study period. Fifth, we used a non-validated modified version of the PACES scale and, therefore, the enjoyment results need to be carefully interpreted. Finally, we did not perform measurements of neurobiological/physiological variables (e.g., 
lactate, cortisol, and serotonin concentrations). Nevertheless, these limitations do not prevent conclusions from being drawn from our results.

\section{Conclusions}

The present study showed that EXG and TC sessions did not reduce state anxiety levels and elicited similar enjoyment and affective valence in healthy young adult men. However, the TC session evoked a higher mean heart rate and better participant training volume than the EXG session. Further studies with clinical populations are needed to expand the results.

Supplementary Information The online version contains supplementary material available at https://doi.org/10.1007/s11332-021-00841-9.

Acknowledgements We would like to thank all the participants who volunteered their time to participate in this study. This study was supported by Coordenação de Aperfeiçoamento de Pessoal de Ensino Superior (CAPES, Brazil; Grant No.: Finance code 001), by Fundação de Amparo à Pesquisa do Estado de Goiás (FAPEG, Brazil; Grant No.: 201210267001056) and by Conselho Nacional de Desenvolvimento Científico e Tecnológico (CNPq, Brazil; Grant No.: 405096/2016-0). WFS, NSM, and TGC were a fellowship from CAPES (Finance code 001). RLV is a productivity fellowship at the Fundação de Amparo à Pesquisa e Inovação do Espírito Santo (FAPES) agency (Edital $N^{\circ}$ 18/2018-Bolsa Pesquisador Capixaba). CABL is a productivity fellowship at the CNPq (Grant No. 305276/2020-4).

Author contributions WFS, NSM and TGC conceived and carried out the screenings and reviews. WFS and RBV carried out the data extraction from articles. WFS carried out the statistical analysis, drafted and revised the manuscript. WFS, RBV, NSM, TGC, RLV, GCTC, MSA, and $\mathrm{CABL}$ revised the manuscript. All authors read and approved the final manuscript.

Funding The authors declare that the research was conducted in the absence of any commercial or financial relationships that could be construed as a potential conflict of interest.

\section{Declarations}

Conflict of interest The authors declare that they have no conflict of interest.

Ethical approval All procedures were approved by the University Research Ethics Committee (approval number 10067119.6.0000.5083) and were in accordance with the principles outlined in the Declaration of Helsinki.

Informed consent All participants were informed about the procedures, and informed consent was obtained before data collection.

Code availability Not applicable.

\section{References}

1. World Health Organization (2017) Depression and other common mental disorders: global health estimates. WHO, Geneva, pp 1-24

2. Goodwin RD (2003) Association between physical activity and mental disorders among adults in the United States. Prev Med (Baltim) 36:698-703. https://doi.org/10.1016/S0091-7435(03) 00042-2

3. Bartley CA, Hay M, Bloch MH (2013) Meta-analysis: aerobic exercise for the treatment of anxiety disorders. Prog Neuro-psychopharmacol Biol psychiatry 45:34-39. https://doi.org/10.1016/j. pnpbp.2013.04.016

4. Jayakody K, Gunadasa S, Hosker C (2014) Exercise for anxiety disorders: systematic review. Br J Sports Med 48:187-196. https:// doi.org/10.1136/bjsports-2012-091287

5. NIMH (2016) Anxiety Disorders. National Institute of Mental Health

6. Puccinelli PJ, da Costa TS, Seffrin A et al (2021) Reduced level of physical activity during COVID-19 pandemic is associated with depression and anxiety levels: an internet-based survey. BMC Public Health. https://doi.org/10.1186/s12889-021-10470-z

7. Wittchen HU, Jacobi F, Rehm J et al (2011) The size and burden of mental disorders and other disorders of the brain in Europe 2010. Eur Neuropsychopharmacol 21:655-679. https://doi.org/10. 1016/j.euroneuro.2011.07.018

8. Ensari I, Greenlee TA, Motl RW, Petruzzello SJ (2015) Metaanalysis of acute exercise effects on state anxiety: an update of randomized controlled trials over the past 25 years. Depress Anxiety 32:624-634. https://doi.org/10.1002/da.22370

9. Gordon BR, McDowell CP, Lyons M, Herring MP (2017) The effects of resistance exercise training on anxiety: a meta-analysis and meta-regression analysis of randomized controlled trials. Sport Med 47:2521-2532. https://doi.org/10.1007/ s40279-017-0769-0

10. Wiemeyer J, Deutsch J, Malone LA et al (2015) Recommendations for the optimal design of exergame interventions for persons with disabilities: challenges, best practices, and future research. Games Health J 4:58-62. https://doi.org/10.1089/g4h.2014.0078

11. Viana RB, Vancini RL, Andrade MS et al (2019) O uso dos exergames nos protocolos de reabilitação em diversas populações clínicas. Rev Bras Pesqui em Saúde 20:132-140

12. Lee S, Kim W, Park T, Peng W (2017) The psychological effects of playing exergames: a systematic review. Cyberpsychol Behav Soc Netw 20:513-532. https://doi.org/10.1089/cyber.2017.0183

13. Morais NS, Viana RB, Silva WF et al (2021) Effect of both dance exergame and a traditional exercise on state anxiety and enjoyment in women. J Sports Med Phys Fitness. https://doi.org/10.23736/ S0022-4707.21.12237-6

14. Guthold R, Stevens GA, Riley LM, Bull FC (2018) Worldwide trends in insufficient physical activity from 2001 to 2016: a pooled analysis of 358 population-based surveys with 1.9 million participants. Lancet Glob Heal 6:e1077-e1086. https://doi.org/10.1016/ S2214-109X(18)30357-7

15. Herazo-Beltrán Y, Pinillos Y, Vidarte J et al (2017) Predictors of perceived barriers to physical activity in the general adult population: a cross-sectional study. Brazil J Phys Ther 21:44-50. https:// doi.org/10.1016/j.bjpt.2016.04.003

16. Viana RB, Vancini RL, Vieira CA et al (2018) Profiling exercise intensity during the exergame hollywood workout on XBOX 360 Kinect. PeerJ. https://doi.org/10.7717/peerj.5574

17. Viana RB, Gentil P, Andrade MS et al (2019) Is the energy expenditure provided by exergames valid? Int J Sports Med. https://doi.org/10.1055/a-0955-9394 
18. Wagener TL, Fedele DA, Mignogna MR et al (2012) Psychological effects of dance-based group exergaming in obese adolescents. Pediatr Obes. https://doi.org/10.1111/j.2047-6310.2012.00065.x

19. Viana RB, Dankel SJ, Loenneke JP et al (2020) The effects of exergames on anxiety levels: a systematic review and meta-analysis. Scand J Med Sci Sports. https://doi.org/10.1111/sms.13654

20. Viana RB, Alves CL, Vieira CA et al (2017) Anxiolytic effects of a single session of the exergame zumba fitness on healthy young women. Games Health J. https://doi.org/10.1089/g4h.2017.0085

21. Graves LEF, Ridgers ND, Atkinson G, Stratton G (2010) The effect of active video gaming on children's physical activity, behavior preferences and body composition. Pediatr Exerc Sci 22:535-546. https://doi.org/10.1123/pes.22.4.535

22. Garber CE, Blissmer B, Deschenes MR et al (2011) American College of Sports Medicine position stand. Quantity and quality of exercise for developing and maintaining cardiorespiratory, musculoskeletal, and neuromotor fitness in apparently healthy adults: guidance for prescribing exercise. Med Sci Sports Exerc. https:// doi.org/10.1249/MSS.0b013e318213fefb

23. Wu PT, Wu WL, Chu IH (2015) Energy expenditure and intensity in healthy young adults during exergaming. Am J Health Behav. https://doi.org/10.5993/AJHB.39.4.12

24. Graves LEF, Ridgers ND, Williams K et al (2010) The physiological cost and enjoyment of Wii fit in adolescents, young adults, and older adults. J Phys Act Health. https://doi.org/10.1123/jpah.7.3. 393

25. Thompson WR (2021) Worldwide survey of fitness trends for 2021. ACSMs Health Fit J 25:10-19. https://doi.org/10.1249/ FIT.0000000000000631

26. Thompson WR (2018) Worldwide survey of fitness trends for 2019. ACSMs Health Fit J 22:10-17. https://doi.org/10.1249/ FIT.0000000000000438

27. Spielberger C, Gorsuch R, Lushene R (1970) Manual for the statetrait anxiety inventory. Consulting Psychologists Press, Palo Alto, CA

28. Spielberger C (1989) State-trait anxiety inventory: Bibliography, 2nd edn. Consulting Psychologists Press, Palo Alto, CA

29. Hower IM, Harper SA, Buford TW (2018) Circadian rhythms, exercise, and cardiovascular health. J Circadian Rhythms. https:// doi.org/10.5334/jcr. 164

30. de Lira CAB, Peixinho-Pena VRL et al (2013) Heart rate response during a simulated Olympic boxing match is predominantly above ventilatory threshold 2: a cross sectional study. Open Access J Sport Med 4:175. https://doi.org/10.2147/OAJSM.S44807

31. Gorenstein C, Andrade L (1996) Validation of a Portuguese version of the Beck depression inventory and the state-trait anxiety inventory in Brazilian subjects. Brazil J Med Biol Res 29:453-457

32. Spielberger C (1989) State-trait anxiety inventory: a comprehensive bibliography. Consulting Psychologists Press, Palo Alto, CA

33. Knight RG, Waal-Manning HJ, Spears GF (1983) Some norms and reliability data for the state-trait anxiety inventory and the zung self-rating depression scale. Br J Clin Psychol 22:245-249. https://doi.org/10.1111/j.2044-8260.1983.tb00610.x

34. Kendzierski D, DeCarlo KJ (1991) Physical activity enjoyment scale: two validation studies. J Sport Exerc Psychol 13:50-64. https://doi.org/10.1123/jsep.13.1.50

35. Alves ED, Panissa VLG, Barros BJ et al (2019) Translation, adaptation, and reproducibility of the physical activity enjoyment scale (PACES) and feeling scale to brazilian portuguese. Sport Sci Health 15:329-336. https://doi.org/10.1007/s11332-018-0516-4

36. Hardy CJ, Rejeski WJ (1989) Not what, but how one feels: the measurement of affect during exercise. J Sport Exerc Psychol 11:304-317. https://doi.org/10.1123/jsep.11.3.304
37. Jung M, Soo Kim H, Loprinzi D (2021) Serial-multiple mediation of enjoyment and intention on the relationship between creativity and physical activity. AIMS Neurosci 8:161-180. https://doi.org/ 10.3934/Neuroscience. 2021008

38. Lenhard W, Lenhard A (2016) Calculation of effect sizes. Psychometrica, Dettelbach (Germany). https://doi.org/10.13140/RG.2.1. 3478.4245

39. Borenstein M, Hedges LV, Higgins JPT, Rothstein HR (2009) Introduction to meta-analysis. Wiley, Chichester

40. Cohen J (1988) Statistical power analysis for the behavioral sciences statistical power analysis for the behavioral sciences, 2nd edn. Lawrence Erlbaum Associates, Hillsdale-New Jersey

41. Rosenthal R (1994) Parametric measures of effect size. In: Cooper $\mathrm{H}$, Hedges LV (eds) The handbook of research synthesis. Russell Sage Foundation, New York, pp 231-244

42. Munro BH (1986) Statistical methods for health care research. JB Lippincott, Philadelphia

43. Anderson E, Shivakumar G (2013) Effects of exercise and physical activity on anxiety. Front Psychiatry 4:1-4. https://doi.org/10. 3389/fpsyt.2013.00027

44. Mullen SP, Olson EA, Phillips SM et al (2011) Measuring enjoyment of physical activity in older adults: invariance of the physical activity enjoyment scale (paces) across groups and time. Int J Behav Nutr Phys Act 8:1-9. https://doi.org/10.1186/ 1479-5868-8-103

45. Hagberg LA, Lindahl B, Nyberg L, Hellénius M-L (2009) Importance of enjoyment when promoting physical exercise. Scand J Med Sci Sports. https://doi.org/10.1111/j.1600-0838.2008. 00844.x

46. Ekkekakis P (2003) Pleasure and displeasure from the body: perspectives from exercise. Cogn Emot 17:213-239. https://doi.org/ 10.1080/02699930302292

47. Rose EA, Parfitt G (2007) A Quantitative analysis and qualitative explanation of the individual differences in affective responses to prescribed and self-selected exercise intensities. J Sport Exerc Psychol 29:281-309. https://doi.org/10.1123/jsep.29.3.281

48. Rose EA, Parfitt G (2008) Can the feeling scale be used to regulate exercise intensity? Med Sci Sport Exerc. https://doi.org/10.1249/ MSS.0b013e31817a8aea

49. Kilpatrick MW, Greeley SJ, Collins LH (2015) The impact of continuous and interval cycle exercise on affect and enjoyment. Res Q Exerc Sport 86:244-251. https://doi.org/10.1080/02701 367.2015.1015673

50. McArdle WD, Katch FI, Katch VL (2014) Exercise physiology: nutrition, energy, and human performance, 8th edn. LWW, Philadelphia

51. Tan B, Aziz AR, Chua K, Teh KC (2002) Aerobic demands of the dance simulation game. Int J Sports Med 23:125-129. https://doi. org/10.1055/s-2002-20132

52. Jordan M, Donne B, Fletcher D (2011) Only lower limb controlled interactive computer gaming enables an effective increase in energy expenditure. Eur J Appl Physiol 111:1465-1472. https:// doi.org/10.1007/s00421-010-1773-3

53. Edwards MK, Loprinzi PD (2016) Experimentally increasing sedentary behavior results in increased anxiety in an active young adult population. J Affect Disord 204:166-173. https://doi.org/ 10.1016/j.jad.2016.06.045

Publisher's Note Springer Nature remains neutral with regard to jurisdictional claims in published maps and institutional affiliations. 